\title{
Gut-liver axis and disease infidelity: A subject worth exploring
}

\author{
Prasenjit Das • Govind K. Makharia
}

Received: 1 September 2014 / Accepted: 1 September 2014 / Published online: 3 October 2014

(C) Indian Society of Gastroenterology 2014

The spectrum of gluten-related disorders is widening and varies from celiac disease $(\mathrm{CeD})$ to non-celiac gluten sensitivity [1]. While small intestine was thought to be the only organ involved in patients with $\mathrm{CeD}$, it is now recognized that the hypersensitivity to gluten is not limited to the small intestine alone and may affect other organs such as skin, brain, and bones independent of intestinal involvement [2-5]. Dermatitis herpetiformis and gluten ataxia are the best example of such phenomena.

In a study published in this issue of the Journal, Maiwall et al. [6] have reported small intestinal changes in 61 patients with cryptogenic chronic liver disease (cryptogenic chronic liver disease 47 and 14 biopsy proven noncirrhotic idiopathic portal hypertension) and 59 patients with hepatitis B or hepatitis $\mathrm{C}$ virus related cirrhosis as controls. All of them were screened for $\mathrm{CeD}$ using anti-tissue transglutaminase antibody (anti-tTG $\mathrm{Ab}$ ) and patients having a positive screening test were invited for upper gastrointestinal endoscopy and duodenal biopsies were obtained from them. $\mathrm{CeD}$ was diagnosed on the basis of combination of a positive serology and presence of villous abnormalities. An unexpectedly high number of both study subjects $(40,66 \%)$ and controls $(17,29 \%)$ were detected to have a positive anti-tTG Ab. Of them, 37 in the study group and 11 in control group underwent duodenal biopsies, respectively. Overall, six patients fulfilled the criteria for the diagnosis of $\mathrm{CeD}$ (including two patients with noncirrhotic intrahepatic portal hypertension [NCIPH]) as compared to none in controls.

\section{P. Das}

Department of Pathology, All India Institute of Medical Sciences, Ansari Nagar, New Delhi 110 029, India

\section{G. K. Makharia $(\square)$}

Department of Gastroenterology and Human Nutrition, All India Institute of Medical Sciences, Ansari Nagar, New Delhi 110 029, India

e-mail: govindmakharia@gmail.com
Additionally, a significant number of both study subject controls also had villous abnormalities (various grade of villous atrophy, crypt hyperplasia, and chronic inflammatory cells in the lamina propria, these changes however were not accompanied by rise in intraepithelial lymphocytes. Such changes were seen more often in cryptogenic cirrhosis than those with cirrhosis due to hepatitis $B$ virus or hepatitis $C$ virus-related cirrhosis. Of patients with negative anti-tTG antibody who underwent duodenal biopsies, 4/16 (25\%) of cases and 4/22 (18\%) of controls also showed the presence of villous abnormalities. Furthermore, after observing a high anti-tTG Ab positive in both study group (cryptogenic cirrhosis) and controls (cirrhosis due to HBV or HCV), the authors retested the serum samples by two different ELISA kits procured from two different manufacturers and showed also remarkable variability in the performance of ELISA kits. The anti-tTG antibody was positive in $16(31 \%)$ cases and in $15(38 \%)$ controls by a kit obtained from Euroimmune and in $12(23 \%)$ cases and 8 (20\%) controls using kits obtained from Inova.

While Maiwall et al. in the present study have shown that approximately $10 \%$ of cryptogenic cirrhosis have coexistent $\mathrm{CeD}$, more importantly, they raise an important question. Is there a causal relationship between $\mathrm{CeD}$ and liver damage, and if so, then how? They also highlighted two another issues. Firstly, that many patients with chronic liver disease (CLD) have some form of enteropathy, irrespective of the cause of cirrhosis. Secondly, there exists a variability in the performance of ELISA done on the same samples using ELISA kits from different manufacturers.

Liver deals with various antigens and pathogens, which evade the intestinal mucosal barrier and reach liver via the portal circulation. Both the small intestine and the liver are equipped with immune systems and are capable of working as a single unit of immunological defense and they cross talk with each other, when needed [7, 8]. This crosstalk between liver and intestine is the backbone of the "Gut-liver" axis and 
is responsible for providing both a physical and immunological wall. Like the hepatorenal axis, the gut-liver axis needs to be recognized because some autoimmune and inflammatory diseases of one organ have the potential of affecting another, symptomatically or silently. While both small and large intestinal diseases such as $\mathrm{CeD}$ and inflammatory bowel disease (IBD) can affect liver as discussed below, liver diseases can also affect the gut.

The spectrum of involvement of liver in patients with $\mathrm{CeD}$ is known to range from asymptomatic elevation of serum transaminases to end-stage liver disease. Elevated serum transaminases have been reported in $24 \%$ to $54 \%$ of patients with $\mathrm{CeD}$ [9-11]. Conversely, up to $9 \%$ of the patients with chronic unexplained increase in transaminases have been reported to have $\mathrm{CeD}$ [12]. Normalization of transaminases in them within 6 months to 1 year of gluten-free diet supports the view that hepatocellular necrosis in these patients is somehow secondary to gluten peptides $[10,11]$. $\mathrm{CeD}$ has also been reported to coexist with other autoimmune liver diseases such as autoimmune hepatitis, primary biliary cirrhosis (PBC), and primary sclerosing cholangitis (PSC). Four percent to $6 \%$ of patients with autoimmune hepatitis are reported to have CeD [11, 13-15]. Similarly, $4 \%$ to $11 \%$ of patients with $\mathrm{PBC}$ have coexistent $\mathrm{CeD}$ [12]. Conversely, patients with $\mathrm{CeD}$ have more than three times increased odds of having $\mathrm{PBC}$ compared to the occurrence of $\mathrm{PBC}$ in the general population $(0.17 \%$ vs. $0.05 \%)$ [16]. Furthermore, patients with $\mathrm{CeD}$ have 4.5 times increased risk of having PSC in comparison to general population as shown by a large population-based cohort study including $13,818 \mathrm{CeD}$ patients [17]. Furthermore, in patients having a combination of both autoimmune liver disorders and $\mathrm{CeD}$, symptoms of one might precede the other, or even may coexist at the time of diagnosis and therefore need careful evaluation [18].

Along with the data presented in the study by Maiwall et al. [6], there are also several levels of evidences that suggest an association between $\mathrm{CeD}$ with CLD or cirrhosis [17, 19-21]. Patients with $\mathrm{CeD}$ are also more likely to die from liver cirrhosis than the general population [21]. Kaukinen et al. had reported reversal of hepatic dysfunction after initiation of gluten-free diet (GFD) in four patients awaiting liver transplantation, and eventually, three of them were remitted from liver transplantation list. Furthermore, CeD was detected in $8(4.3 \%)$ of 185 patients who underwent liver transplantation previously and later screened for $\mathrm{CeD}$ [22]. In a more recent prospective study, Wakim-Fleming et al. have reported $\mathrm{CeD}$ in $2.5 \%$ of 204 consecutive patients with biopsy-proven liver cirrhosis [20]. They also demonstrated improvement in their liver function tests with initiation of GFD in them [20].

Not only in CeD, liver gets affected in IBD too. Two percent to $8 \%$ patients with IBD show the presence of autoimmune hepatitis, PSC or overlap syndrome. Moreover, $70 \%$ to $80 \%$ patients with PSC show synchronous or metachronous IBD [23].

It is now recognized that the alteration in immune functions, both in IBD and CeD, not only affect the epithelium, but also the blood vessels in the liver and other organs, leading to thrombotic and thromboembolic manifestations. The local vasculature connecting these two organs such as portal vein, hepatic artery, and their branches are likely to be the site of main involvement in such conditions [24]. While both $\mathrm{CeD}$ and noncirrhotic portal fibrosis can coexist in a patient independent of each other, the co-occurrence of these two diseases, as in the present study, and a few previous studies, raises a question if there is a causal relationship between these two disorders. One can hypothesize, that the endothelial injury and hypercoagulable state in some patients with $\mathrm{CeD}$ may lead to microvasculature block/injury causing noncirrhotic idiopathic portal hypertension and major vasculature block causing Budd-Chiari syndrome, which already have been described to coexist with $\mathrm{CeD}$ at least in some patients [25-28].

The abovementioned evidences while suggest an association between $\mathrm{CeD}$ and spectrum of liver diseases, morphological changes that occur in the liver however is not well known except for a study by Jabobson et al. who have reported histological changes in the liver of 37 patients with $\mathrm{CeD}$ having increase in serum transaminases [29]. Of 37 of these patients, 5 had normal liver on histological examination, 25 had nonspecific necroinflammatory changes in the liver, 6 had chronic hepatitis, and one had PSC [20].

How exactly immunogenic peptides of gluten lead to liver dysfunction/damage is not known. While population-based studies support an association between autoimmune hepatitis, PSC, and $\mathrm{PBC}$ with $\mathrm{CeD}$, the exact reasons of association between them is not well established. While it is well known that patient having one autoimmune disease can have another autoimmune disease, therefore, mere presence of two autoimmune diseases in an individual do not confirm causality. The immune mechanism of the gut-liver axis act differently in physiological and pathological conditions. In physiological conditions, the antigens pass through the $\mathrm{M}$ cells over the ileal Peyer's patches and are then transported to the mucosal lymphoid follicles or the mesenteric lymph nodes, where the lymphocytes are presented these antigens to T-cells. These activated lymphocytes then express various receptors, as chemokine receptor CCR9, integrin $\alpha 4 \beta 7$, or vascular adhesion protein-1 (VAP-1) [8, 30, 31]. These lymphocytes undergo pre-programmed specific homing either to the intestine or liver, based on the specific ligands expressed in the organs for these receptors. For example, while CCL25 and mucosal addressin cell adhesion molecule 1 (MAdCAM-1) are expressed in the gut endothelial cells, VAP is expressed in 
hepatic endothelial cells. CCR9 and integrin $\alpha 4 \beta 7$ receptor containing lymphocytes are attracted toward ligands CCL25 and MAdCAM-1 in the intestine, while VAP-1 binds to the liver VAP [8, 30, 31]. Such a well organized trafficking fails in many inflammatory and autoimmune diseases as such as $\mathrm{CeD}$ and IBD, where MAdCAM-1, CCL25, or VAP-1 are expressed non-selectively on both the intestine and the liver. The co-occurrence of $\mathrm{CeD}$ and autoimmune hepatitis can also be linked through the sharing of class II HLA molecules encoding for HLA complex genes on chromosome 6 [10]. Furthermore, the entry of antigen also gets aggravated due to the increased leakiness of the gut both in the active $\mathrm{CeD}$ and cirrhosis of the liver. This leads to simultaneous homing of immune reactive lymphocytes to both of these organs and while executing their function, these immune reactive cells have potential for damaging both the organs [32]. Apart from selective homing during the physiological state immune tolerance of the liver and biliary tree prevent an unwanted inflammatory reaction.

At present, there is no tissue specific marker/staining/ cellular repertoire to support liver damage caused by gluten peptide. One of the valid requirements for establishing the association between liver disease and $\mathrm{CeD}$ is to demonstrate reversibility of the liver damage by gluten-free diet. Further studies are needed to better delineate the pathogenesis of liver involvement in CeD. Also, long-term follow up and paired liver biopsies in patients with coexistent $\mathrm{CeD}$ and liver diseases, while being on strict GFD, would help in delineating the mechanisms of liver damage in patients with $\mathrm{CeD}$.

Which are the patients with CLD who should be investigated for presence of concomitant CeD? The reasons for suspicion of $\mathrm{CeD}$ in patients with cirrhosis generally are presence of chronic diarrhea, short stature, and disproportionately severe anemia or iron refractory anemia in them [18, 19]. Chronic diarrhea in patients with cirrhosis may be caused by other causes such as bacterial overgrowth and intestinal mucosal edema. Similarly, anemia in cirrhotic patients could be caused by gastrointestinal bleeding, hypersplenism and anemia of chronic disease. The stunting of growth can well be explained if liver disease sets in early years of life. Therefore, most of these manifestations pointing toward presence of $\mathrm{CeD}$ in a patient with $\mathrm{CeD}$ can well be explained by cirrhosis itself and thus alternative causes for such manifestations may not be considered in a patient with cirrhosis in the particular clinical setting. Furthermore, as shown in the study by Maiwall et al. [6], enteropathic changes were seen in large number of patients with cirrhosis; they however lacked increase in intraepithelial lymphocytes, which are the essential pathological factor feature associated with $\mathrm{CeD}$. Because of overlapping manifestions of cirrhosis and $\mathrm{CeD}$, there is a likelihood of missing the diagnosis of $\mathrm{CeD}$ unless actively considered, if the main presentation of $\mathrm{CeD}$ is like cirrhosis.
The study by Maiwall et al. [6] while has many limitations, it however has brought some extremely important facts as described above. A few limitations in the study by Maiwall et al. [6] necessitates a mention. Firstly, the authors have clubbed the two different diseases of the liver, such as cryptogenic cirrhosis and NCIPH, into one. Furthermore, only a few in the so-called cryptogenic cirrhosis underwent liver biopsy, therefore, one cannot be sure how many of them had NCIPH, if all cryptogenic liver diseases had undergone liver biopsies. Secondly, because of the variability in the performance of anti-tTG Ab estimation by ELISA, authors could have used anti-endomysial antibody test also. Furthermore, it will be good to follow up all the patients with cirrhosis and $\mathrm{CeD}$, especially those six patients who had liver biopsies at the beginning of the gluten-free diet and then follow them up after couple of years for any histological resolution with gluten-free diet. Demonstration of histological resolution of liver lesions with gluten-free diet will provide stronger evidence to support an association between $\mathrm{CeD}$ and liver diseases.

\section{References}

1. Ludvigsson JF, Leffler DA, Bai JC, et al. The Oslo definitions for coeliac disease and related terms. Gut. 2013;62:43-52.

2. Hadjivassiliou M, Sanders DS, Grünewald RA, Woodroofe N, Boscolo S, Aeschlimann D. Gluten sensitivity: from gut to brain. Lancet Neurol. 2010;9:318-30.

3. Antiga E, Caproni M, Pierini I, Bonciani D, Fabbri P. Gluten-free diet in patients with dermatitis herpetiformis: not only a matter of skin. Arch Dermatol. 2011;147:988-9.

4. Marsh MN. Gluten, major histocompatibility complex, and the small intestine. A molecular and immunobiologic approach to the spectrum of gluten sensitivity ('celiac sprue'). Gastroenterology. 1992;102: 330-54.

5. Makharia G. Where are Indian adult celiacs? Trop Gastroenterol. 2006;27:1-3.

6. Maiwall R, Goel A, Pulimood AB, et al. Investigation into celiac disease in Indian patients with portal hypertension. Indian $\mathrm{J}$ Gastroenterol. 2014;33:doi:10.1007/s12664-014-0501-z.

7. Trivedi PJ, Adams DH. The hepato-enteric immune axis in health and disease. Clinic Med. 2012;12:s74-8.

8. Adams DH, Eksteen B, Curbishley SM. Immunology of the gut and liver: a love/hate relationship. Gut. 2008;57:838-48.

9. Sharma M, Singh P, Agnihotri A, et al. Celiac disease: a disease with varied manifestations in adults and adolescents. J Dig Dis. 2013;14: 518-25.

10. Rostami-Nejad M, Haldane T, Aldulaimi D, Alavian SM, Zali MR, Rostami K. The role of celiac disease in severity of liver disorders and effect of a gluten free diet on diseases improvement. Hepat Mon. 2013;13:e11893.

11. Rubio-Tapia A, Murray JA. The liver in celiac disease. Hepatol Baltim Md. 2007;46:1650-8.

12. Volta U, De Franceschi L, Lari F, Molinaro N, Zoli M, Bianchi FB. Coeliac disease hidden by cryptogenic hypertransaminasaemia. Lancet. 1998:352:26-9.

13. Volta U, De Franceschi L, Molinaro N, et al. Frequency and significance of anti-gliadin and anti-endomysial antibodies in autoimmune hepatitis. Dig Dis Sci. 1998;43:2190-5. 
14. Villalta D, Girolami D, Bidoli E, et al. High prevalence of celiac disease in autoimmune hepatitis detected by anti-tissue tranglutaminase autoantibodies. J Clin Lab Anal. 2005;19:6-10.

15. van Gerven NM, Bakker SF, de Boer YS, et al. On behalf of the Dutch AIH working group. Seroprevalence of celiac disease in patients with autoimmune hepatitis. Eur J Gastroenterol Hepatol. 2014;26:1104-7.

16. Sorensen HT, Thulstrup AM, Blomqvist P, Nørgaard B, Fonager K, Ekbom A. Risk of primary biliary liver cirrhosis in patients with coeliac disease: Danish and Swedish cohort data. Gut. 1999;44:736-8.

17. Ludvigsson JF, Elfström P, Broomé U, Ekbom A, Montgomery SM. Celiac disease and risk of liver disease: a general population-based study. Clin Gastroenterol Hepatol. 2007;5:63-9.

18. Caprai S, Vajro P, Ventura A, Sciveres M, Maggiore G, SIGENP Study Group for Autoimmune Liver Disorders in Celiac Disease. Autoimmune liver disease associated with celiac disease in childhood: a multicenter study. Clin Gastroenterol Hepatol. 2008;6:803-6.

19. Singh P, Agnihotri A, Jindal G, et al. Celiac disease and chronic liver disease: is there a relationship? Indian $\mathrm{J}$ Gastroenterol. 2013;32:404-8

20. Wakim-Fleming J, Pagadala MR, McCullough AJ, et al. Prevalence of celiac disease in cirrhosis and outcome of cirrhosis on a gluten free diet: a prospective study. J Hepatol. 2014;61:558-63.

21. Lindgren S, Sjöberg K, Eriksson S. Unsuspected coeliac disease in chronic "cryptogenic" liver disease. Scand J Gastroenterol. 1994;29: $661-4$.
22. Kaukinen K, Halme L, Collin P, et al. Celiac disease in patients with severe liver disease: gluten-free diet may reverse hepatic failure. Gastroenterology. 2002;122:881-8.

23. Talwalkar JA, Lindor KD. Primary sclerosing cholangitis. Inflamm Bowel Dis. 2005;11:62-72.

24. Halfdanarson TR, Litzow MR, Murray JA. Hematologic manifestations of celiac disease. Blood. 2007;109:412-21.

25. Kochhar R, Masoodi I, Dutta U, et al. Celiac disease and Budd-Chiari syndrome: report of a case with review of literature. Eur $\mathrm{J}$ Gastroenterol Hepatol. 2009;21:1092-4.

26. Hmami F, Chaouki S, Souilmi FZ, et al. Association of celiac disease and Budd-Chiari syndrome: a case report. Arch Pediatr. 2011;18:102.

27. Zamani F, Amiri A, Shakeri R, Zare A, Mohamadnejad M. Celiac disease as a potential cause of idiopathic portal hypertension: a case report. J Medical Case Reports. 2009;3:68.

28. Sharma BC, Bhasin DK, Nada R. Association of celiac disease with non-cirrhotic portal fibrosis. J Gastroenterol Hepatol. 2006;21:332-4.

29. Jacobsen MB, Fausa O, Elgjo K, Schrumpf E. Hepatic lesions in adult coeliac disease. Scand J Gastroenterol. 1990;25:656-62.

30. Wurbel MA, McIntire MG, Dwyer P, Fiebiger E. CCL25/CCR9 interactions regulate large intestinal inflammation in a murine model of acute colitis. PLoS One. 2011;6:e16442.

31. Oo YH, Adams DH. The role of chemokines in the recruitment of lymphocytes to the liver. J Autoimmun. 2010;34:45-54.

32. Adams DH, Eksteen B. Aberrant homing of mucosal $\mathrm{T}$ cells and extraintestinal manifestations of inflammatory bowel disease. Nat Rev Immunol. 2006;6:244-51. 